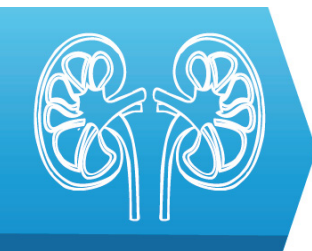

UROLOGY
1) Institute of Urology and Renal Transplantation, Cluj-Napoca, Romania

2) Iuliu Hatieganu University of Medicine and Pharmacy, Cluj-Napoca, Romania

3) CF Clinical Hospital, Cluj-Napoca, Romania

4) Department of Medical Imaging, Iuliu Hatieganu University of Medicine and Pharmacy, "Prof. Dr. Octavian Fodor", Regional Institute of Gastroenterology and Hepatology ClujNapoca, Romania
DOI: $10.15386 / \mathrm{mpr}-1293$

Manuscript received: 29.01.2019

Received in revised form: 14.02 .2020

Accepted: 13.03.2020

Address for correspondence:

nucu.marica@gmail.com

This work is licensed under a Creative Commons Attribution-NonCommercialNoDerivatives 4.0 International License

\title{
Embryonal carcinoma of an intraabdominal testicular tumor on an undescended testicle: a case report
}

Mihai Domnutiu Suciu ${ }^{1,2}$, Nucu Alexandru Marica ${ }^{1,2}$, Traian Oniu ${ }^{3}$, Andrei Ionut Tomuta ${ }^{1}$, Radu Badea ${ }^{4}$

\begin{abstract}
Cryptorchidism, as a singular pathology or associated with other dysgenesis syndromes, is one of the main factors of risk for the development of the testicular tumors. Although there are a great number of cases of undescended testicles that are diagnosed and treated during the first 6-12 months of life, there are rare cases of adults who are undiagnosed and untreated from this anomaly, which can present a high risk of malignancy.

In this study we present the case of a 36-year-old patient, diagnosed at puberty with left cryptorchidism, untreated, who also hadevidenced a large intraabdominal tumoral mass associated with it. The tumoral mass had its origin in the undescended left testicle. Surgical excision of the tumor and retroperitoneal lymphadenectomy was performed. The histological result revealed embryonal carcinoma, without lymphnode metastasis.
\end{abstract}

Adult patients with untreated cryptorchidism should be thoroughly investigated, as they have a high risk of developing testicular cancer.

Keywords: cryptorchidism, embryonal carcinoma, abdominal cavity, testicular neoplasms

\section{Introduction}

$5 \%$ of all urological tumors and $1 \%$ of all cancers found in the global male population are represented by testicular neoplasms, while in Western Europe the age-standardized incidence has been reported to be even higher $(7.8 \%)[1,2]$. Seminomas are most often diagnosed in the 25- to 40-year-old age group, whereas nonseminomatous tumors occur in even younger men (adolescence to 30 years) $[3,4]$.

Cryptorchidism represents the absence of a testicle in the scrotum. The classic definition of the ectopic testicle is the following: "the truly cryptorchid testis is the testis that lies above the external inguinal ring, either within the inguinal canal or within the abdomen, and it is nonpalpable in the unanesthetized patient" [5]. It occurs when there is a congenital defect in the embryological process of testicular descent [6]. Cryptorchidism increases the risk of malignant transformation up 5-10 times compared to the normal population [7]. Because of the improved diagnostic techniques available today and methodical performing of orchidopexy in the infant period, cases of testicular tumors formed on undescended testis are rarely seen and reported in the urologic field.

\section{Case report}

We present the case of a 36-yearold male patient, of urban origin, who was admitted to hospital with left iliac fossa pain and fatigue. The patient had been diagnosed with left cryptorchidism by his primary care physician at puberty, although he had not been investigated further and no warnings had been raised regarding his increased malignancy risk. He has 2 children and an active sexual life. He was not known with any other malignancy or disease. Clinical examination revealed that 
the left testicle was not palpable in the left hemiscrotum, nor in the inguinal canal; palpation of the abdomen revealed a tumoral mass in the left iliac fossa. There was no palpable supraclavicular lymphnodes. Blood test results were normal (WBCs $5 \times 10^{9} / \mathrm{L}, \mathrm{Hgb} 15 \mathrm{~g} / \mathrm{dL}$, blood creatinine $0.8 \mathrm{mg} /$ $\mathrm{dL}$ ), while serum tumoral markers were elevated: Alphafetoprotein $(258 \mathrm{U} / \mathrm{I})$, human chorionic gonadotrophin hCG $(15000 \mathrm{mIU} / \mathrm{ml})$, Lactate dehydrogenase (507 U/I).

Scrotal intravenous CEUS (Contrast-Enhanced Ultrasound) using SonoVue ${ }^{\mathrm{TM}}$ described only the right testicle in the scrotum, which was formed normally, while abdominal ultrasound demonstrated a pelvic tumor, $11 \times 10 \times 7 \mathrm{~cm}$, with a well defined, inhomogeneous structure, hyperenhancing from periphery to the center (Figure 1).

Abdominal contrast-enhanced computed tomography raised concerns over tumoral invasion in the anterior rectal wall (Figure 2), but the MRI described an incapsulated tumor, in contact with the rectum, sigma, bladder, seminal vesicles and ileus (Figure 3). Enlarged left paraaortic lymphnodes were also discovered.

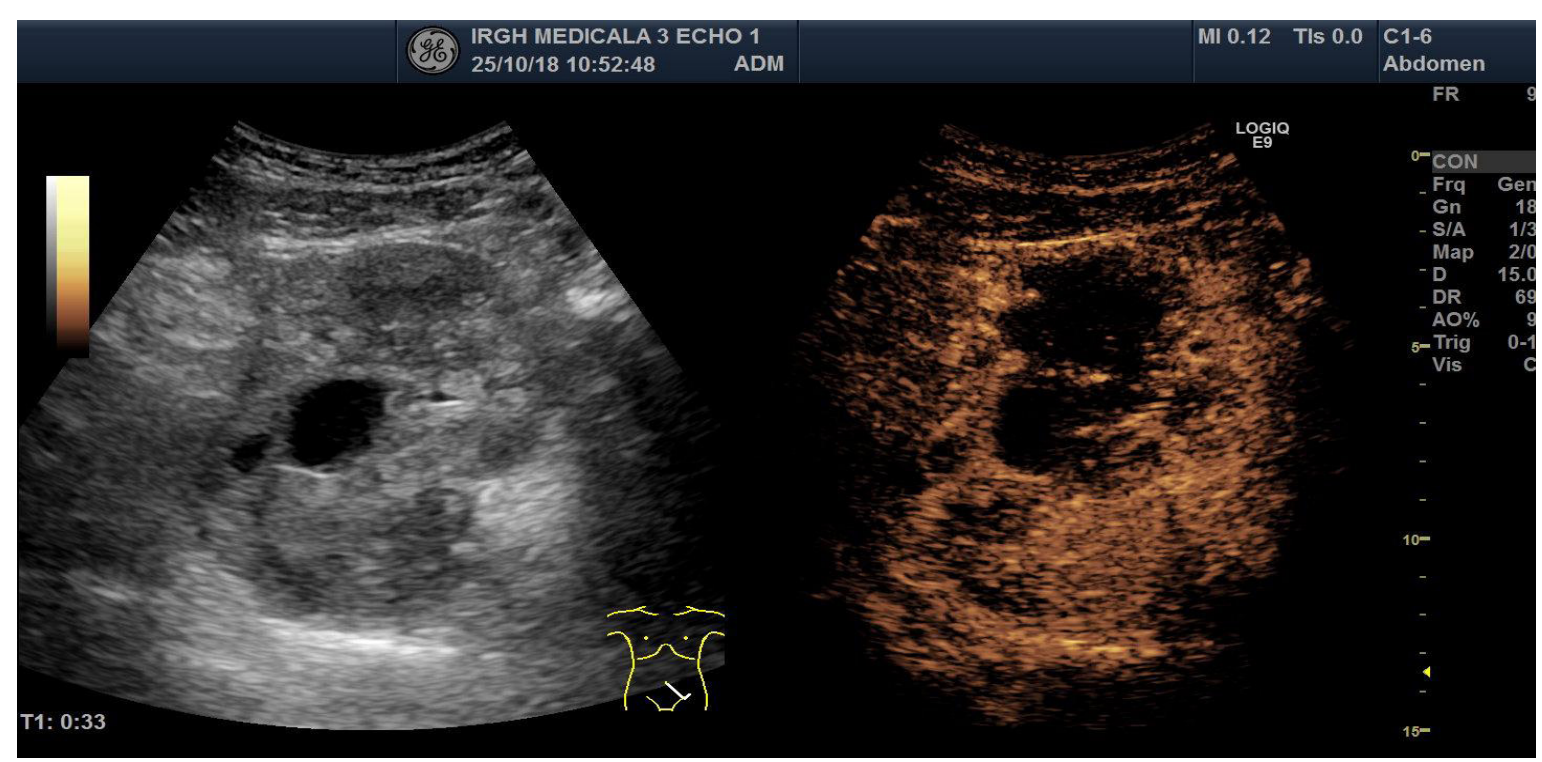

Figure 1. Abdominal ultrasonography performed in standard and SonoVue-enhanced mode.

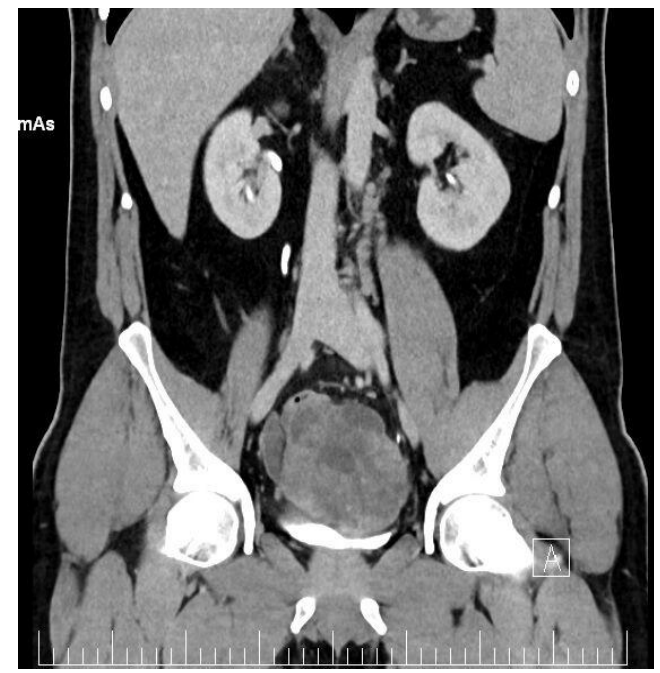

Figure 2. Computed tomography (CT) - coronal plane.

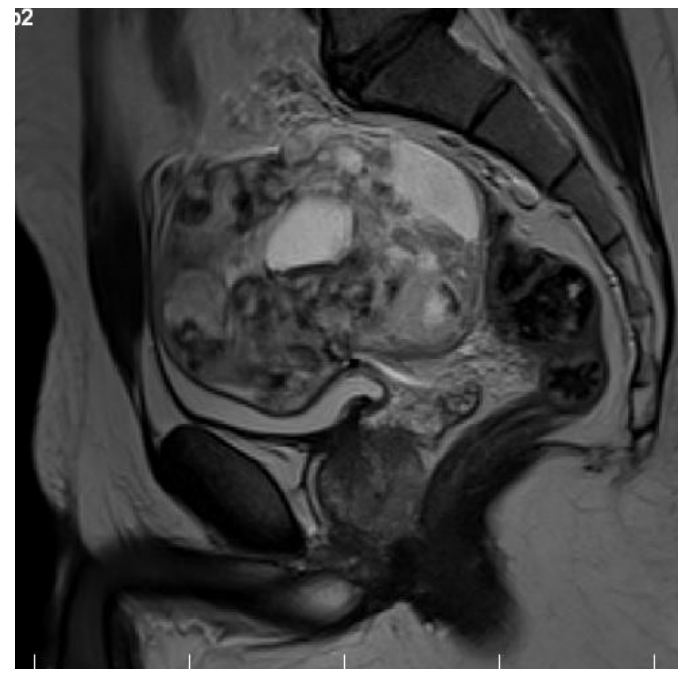

Figure 3. Magnetic resonance imaging (MRI) - sagittal plane. 
Surgical exploration through laparotomy was performed and it revealed a large retroperitoneal tumor $(330 \mathrm{~mL})$ (Figure 4), well defined, without invasion of the nearby organs, and enlarged left paraaortic lymphnodes (Figure 5).

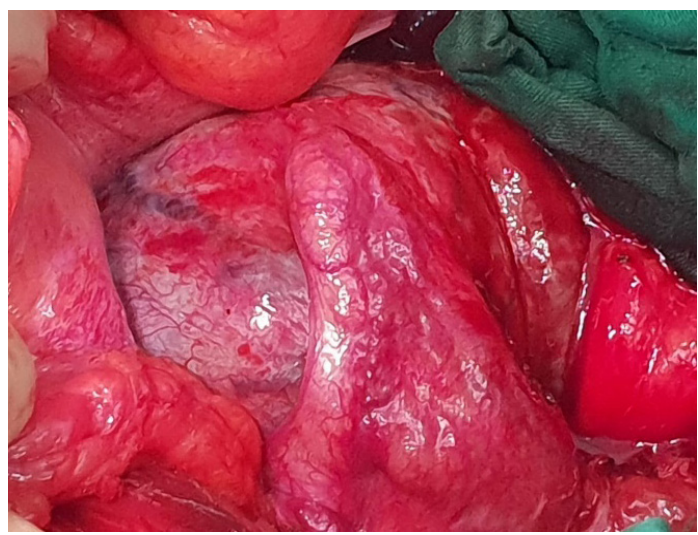

Figure 4. Intraoperative aspect of the tumor.

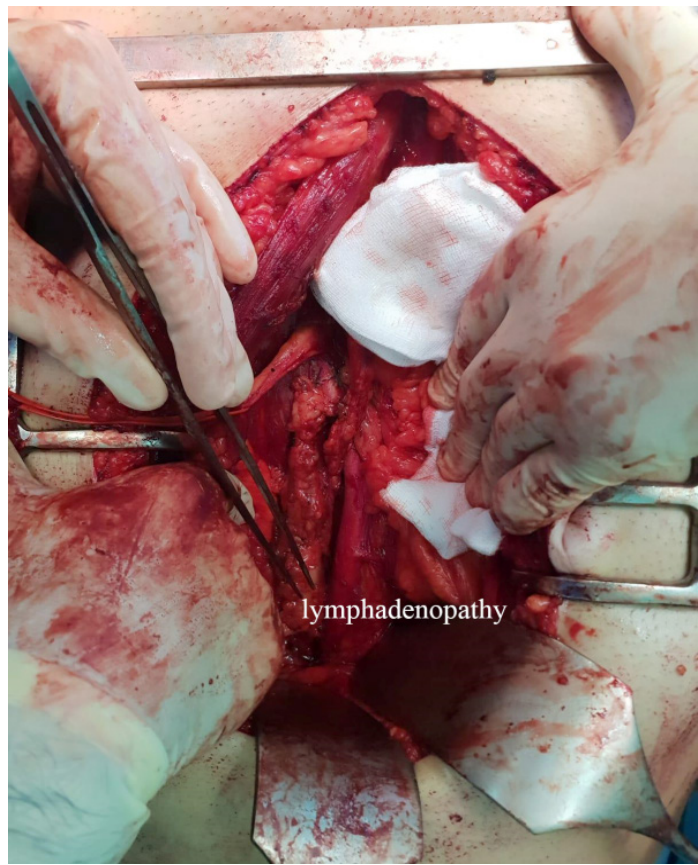

Figure 5. Enlarged paraaortic lymphnodes.

Tumor excision and modified left template retroperitoneal lymphadenectomy (RPLND) were performed (Figure 6). There were no intraoperative or postoperative complications. The patient received antibiotic treatment with Ceftriaxone and pain medication. The urinary catheter was removed the following day and the patient was discharged after 3 days.

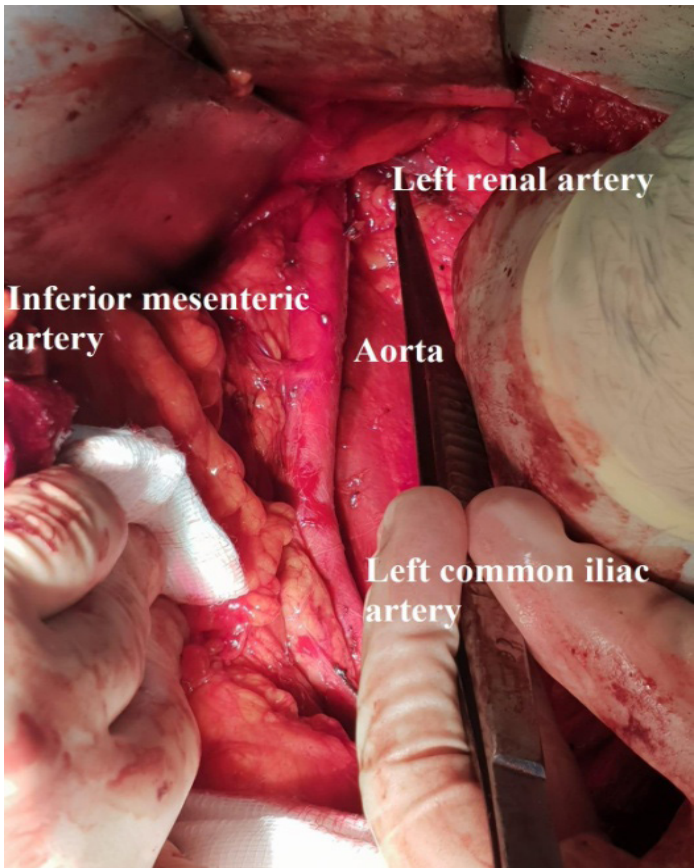

Figure 6. Left retroperitoneal lymph node dissection.

Histopathological examination of the retroperitoneal mass showed pure embryonal carcinoma (Figure 7). The tumor had a solid pattern, composed of primitive epithelial type cells, with minimal features of differentiation. There were mainly high grade features consisting of large, epithelioid cells with prominent nucleoli, indistinct cell borders with nuclear overlapping, pleomorphism, frequent mitoses and with important syncytiotrophoblastic components (Figure 8). Necrosis and intratumoral hemorrhage was present. All excised lymphnodes contained histiocytosis, but without tumoral invasion.

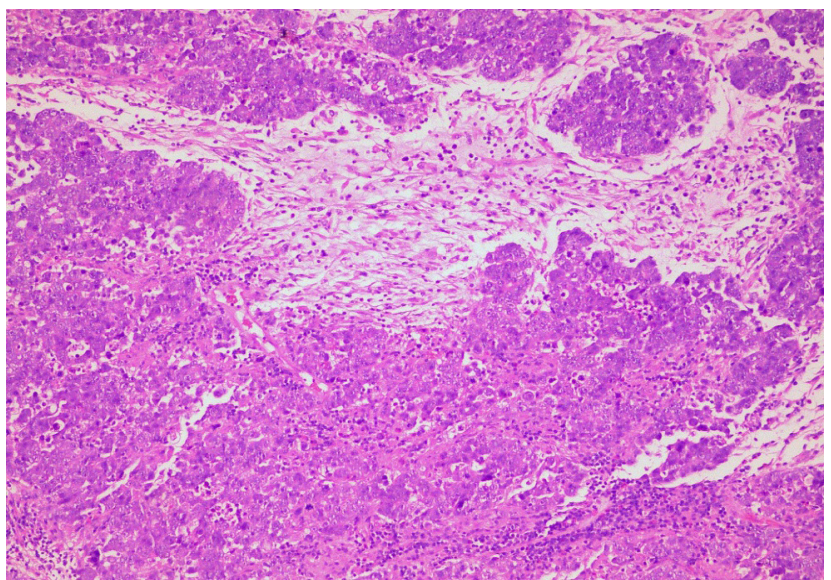

Figure 7. Embryonal cell carcinoma, H\&E stain 10x. 


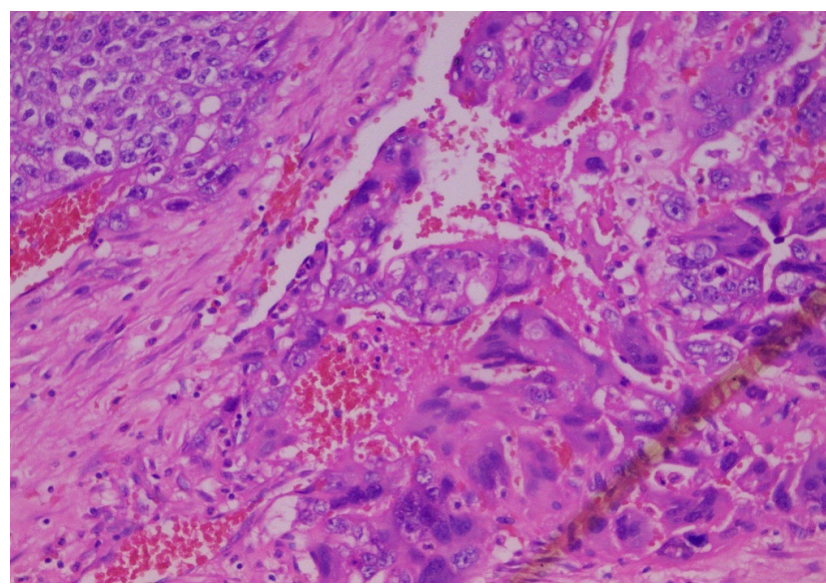

Figure 8. Syncytiotrophoblast component, H\&E stain 20x.

The patient was additionally assessed by chest CT which showed multiple right pulmonary nodules of 10-12 mm. His stadialization was embryonal carcinoma pT2N0M1(a)S2 stage group IIIB, IGCCCG (International Germ Cell Cancer Collaborative Group) intermediateprognosis group. He was sent for oncological surveillance where, based on his prognostic group and ECOG Performance Status of 0 (Eastern Cooperative Oncology Group), he followed adjuvant chemotherapy (4 cycles of BEP - bleomycin, etoposide, cisplatin) with serum markers regression within normal limits after the first cycle, thus being considered chemotherapy-responsive. At the end of the 4 cycles, a follow-up chest and abdominopelvic CT was made which did not show any metastases. Taking into account the RECIST 1.1 criteria [8] the case was considered a complete response. A PET-CT was performed as a 6 months follow-up, without any metastases found, and serum markers in normal limits. The follow-up scheme after complete remission consists of serum markers and doctor visit every 3 months for the first two years and every six months until the $5^{\text {th }}$ year, abdominopelvic and chest computed tomography at 12 months, 24 months, 36 months and 60 months.

\section{Discussion}

Cryptorchidism affects $2 \%-4 \%$ of term male infants and up to one third of premature male newborns, thus it can be considered a common pathology [9]. It occurs when the testicle fails to descend from the lumbar region to the scrotum during natural migration. Long term cryptorchidism can lead to infertility, hormonal changes and neoplastic degeneration [10]. The higher the region of testicle descent is (inguinal, abdominal) the higher the risk of malignancy [11]. The recommended age for orchidopexy has fallen progressively over the last 50 years, from adolescence in the 50 s and 60 s to $6-12$ months in recent times [5]. Since the testicle may descend spontaneously during the first months of life, orchidopexy should not be performed in the first 6 months [12], and ideally before 12 months, in order to optimize fertility outcomes [13]. Concerning testicular malignancy, it has been stated that that there is a relative risk between 2 and 3 noted in patients who have undergone orchidopexy by ages 10 to 12 years, while patients with no orchidopexy, or performed after the age of 12, are 2-6 times more likely to have testicular cancer $[14,15]$.

Regarding the management of undescended testis, the RR of malignant pathology in undescended testes is 40 times higher than in descended ones. Taking into account the mortality rate due to cancer and surgical risks associated with orchiectomy, earlier studies (Farrer- 1985) have suggested the age of 32 should be the age limit for orchiectomy in undescended testicles [16]. From this point of view, a study stratified the anesthetic risk by ASA classification and recommended performing orchiectomy up to 50 years in ASA I and II patients, and it may also be considered in the case of healthy ASA I for patients up to 60 years [17].

Out of all the malignant tumors developed from uncorrected abdominal or inguinal testes, 74\% are seminoma, while corrected cryptorchid or scrotal testicles that undergo malignant transformation are most likely to become nonseminomatous (63\%), presumably because of a decreased risk of seminoma [18].

While we used abdominopelvic computed tomography for diagnostic purposes, it was also useful for staging purposes, as it is known that it has a sensitivity of $70-80 \%$ in assessing retroperitoneal metastatic nodes [19]. In cases of testicular tumors, the European Association of Urology states in its dedicated guideline a strong recommendation that chest $\mathrm{CT}$ is performed in all patients [1]. It has been previously reported that one in ten patients can present minor subpleural nodes that are not seen on X-ray [20]. Computed tomography or MRI of the brain is indicated in the case of coexistence of non-seminomatous germ cell tumors, pulmonary metastases and a poorprognosis International Germ Cell Cancer Collaborative Group (IGCCG) risk group - values of elevated betahCG [1]. Although there is also a recommendation to perform fertility investigations - semen analysis, Folliclestimulating hormone (FSH), Luteinizing hormone (LH), total testosterone - they were not performed.

Embryonal carcinoma is one of the most common components in mixed germ cell tumors, while pure embryonal carcinoma only occurs in $3-4 \%$ of the cases [21], usually occurring in the second and third decade of life [22]. It is an aggressive tumor associated with a high rate of metastasis while tumoral markers are often at normal levels. Embryonal carcinoma is known to have the highest probability of lymphatic and vascular invasion and also of extension into the paratesticular tissue [23], as it also has the tendency to early hematogenous dissemination. Patients diagnosed with embryonal carcinoma usually have metastatic disease, and half of them have distant metastases 
[24]. Mediastinal primary tumor, nonpulmonary visceral metastasis or post-orchiectomy markers any of: AFP > $10,000 \mathrm{ng} / \mathrm{mL}$, beta-hCG $>50,000 \mathrm{iu} / \mathrm{L}, \mathrm{LDH}>10 \times$ upper limit of normal, are considered unfavourable prognostic factors for non-seminoma [25]. Patients with IGCCG intermediate-prognosis group are considered to have a 5 -year progression-free survival of $75 \%$ and 5 -year survival of $80 \%$ [26].

Contrast-enhanced ultrasound (CEUS) is the application of ultrasound contrast medium to traditional ultrasonography. Although conventional grayscale ultrasonography and color Doppler ultrasonography are considered gold standard techniques for evaluating scrotal masses [27], recent studies have pointed out the advantages of complementary CEUS in characterizing testicular neoplasms [28,29]. SonoVue (Bracco SpA, Milan, Italy), which is considered one of the most frequently used contrast agents in (CEUS), is a second-generation contrast agent that uses sulphur hexafluoride microbubbles for CEUS imaging in adults, enhancing blood echogenicity and improving the signal-to-noise ratio in ultrasonography [30]. In a prospective study of 68 patients where CEUS, real-time elastography, B-mode and color-coded Doppler sonography of scrotal tumors were performed, Schröder et al. recommend using CEUS in small testicular masses, where it is impossible to differentiate between malignant and benign lesions, given the fact that it can indicate a neoplasm by recognizing a previously undetected hypervascularization, thus impacting the treatment strategy [31].

Germ cell neoplasia in situ (GCNIS) has an incidence of $9 \%$, while metachronous testicular tumors have approx. $2.5 \%$. Thus, the EAU guidelines have a strong recommendation to perform biopsy of the contralateral testis in patients with a high risk of contralateral GCNIS, consisting of having the volume of testicle less than $12 \mathrm{~mL}$, inferior spermatogenesis or a history of cryptorchidism [1]. Newer studies have shown that microRNA371a-3p could guide in better selecting patients that require surgical biopsies and replace control biopsies following the treatment of germ cell neoplasia in situ [32]. Another strong recommendation is concerning the family members of the patient, who should perform scrotal self-examination in a regular manner, just as other populations at risk should $[1,33]$.

\section{Conclusion}

Adults patients diagnosed with cryptorchidism should be carefully investigated, as there is a greater probability of malignancy. Although both orchidopexy and orchidectomy can be performed, depending on the age, patients should be informed about life-long malignancy risks. Still, cases of testicular tumors developed at this level are rare, for instance pure embryonal intraabdominal testicular cancer, but early treatment by surgery and combined with chemotherapy administration can lead to significant cure rates. Intravenous CEUS can be used for assessing an intraabdominal testicular tumor, although additional imaging is necessary. This case emphasizes the need of performing the diagnosis and treatment of cryptorchidism as early as possible and raising awareness of the importance of testicular self - examination.

\section{References}

1. EAU Guidelines. Edn. presented at the EAU Annual Congress Barcelona 2019. ISBN 978-94-92671-04-2.

2. Rosen A, Jayram G, Drazer M, Eggener SE. Global trends in testicular cancer incidence and mortality. Eur Urol. 2011;60:374-379.

3. Garner MJ, Turner MC, Ghadirian P, Krewski D. Epidemiology of testicular cancer: an overview. Int J Cancer. 2005;116:331-339.

4. Moch H, Humphrey PA, Ulbright TM, Reuter VE, (Eds). WHO Classification of Tumours of the Urinary System and Male Genital Organs, $4^{\text {th }}$ Edition. IARC Press, Lyon 2016.

5. Sheldon CA. Undescended testis and testicular torsion. Surg Clin North Am. 1985;65:1303-1329.

6. Hutson JM, Thorup JM, Beasley SW. Inguinoscrotal Descent of the Testis. In: Descent of the Testis. Springer, Cham; 2016: pp. 29-44.

7. United Kingdom Testicular Cancer Study Group. Aetiology of testicular cancer: Association with congenital abnormalities, age at puberty, infertility, and exercise. $\mathrm{Br}$ Med J. 2008;308:1393-1399.

8. Schwartz LH, Litière S, de Vries E, Ford R, Gwyther S, Mandrekar S, et al. RECIST 1.1-Update and clarification: From the RECIST committee. Eur J Cancer. 2016;62:132-137.

9. Cortes D, Kjellberg EM, Breddam M, Thorup J. The true incidence of cryptorchidism in Denmark. J Urol. 2008;179:314-318.

10. La Vignera S, Calogero AE, Condorelli R, Marziani A, Cannizzaro MA, Lanzafame F, et al. Cryptorchidism and its long-term complications. Eur Rev Med Pharmacol Sci. 2009; 13:351-356.

11. Giwercman A, Bruun E, Frimotd-Møller C, Skakkebaek NE. Prevalence of carcinoma in situ and other histopathological abnormalities in testes of men with a history of cryptorchidism. J Urol. 1989;142:998-1001: discussion 1001-1002.

12. Wenzler DL, Bloom DA, Park JM. What is the rate of spontaneous testicular descent in infants with cryptorchidism? J Urol. 2004;171(2 Pt 1):849-851.

13. Ritzén EM, Bergh A, Bjerknes R. Christiansen P, Cortes D, Haugen SE, et al. Nordic consensus on treatment of undescended testes. Acta Paediatr. 2007;96:638-643.

14. Chan E, Wayne C, Nasr A; FRCSC for Canadian Association of Pediatric Surgeon Evidence-Based Resource. Ideal timing of orchiopexy: a systematic review. Pedriatr Surg Int. 2013;30:87-97.

15. Wood HM, Elder JS. Cryptorchidism and testicular cancer: separating fact from fiction. J Urol. 2009;181:452-461. 
16. Farrer JH, Walker AH, Rajfer J. Management of the postpubertal cryptorchid testis: a statistical review. J Urol 1985;134: 1071-1076.

17. Oh J, Landman J, Evers A, Yan Y, Kibel AS. Management of the postpubertal patient with cryptorchidism: an updated analysis. J Urol. 2002;167:1329-1333.

18. Wood HM, Elder JS. Cryptorchidism and testicular cancer: separating fact from fiction. J Urol. 2009;181:452-461.

19. Smith ZL, Werntz RP, Eggener SE. Testicular Cancer: Epidemiology, Diagnosis, and Management. Med Clin North Am. 2018;102:251-264.

20. See WA, Hoxie L. Chest staging in testis cancer patients: imaging modality selection based upon risk assessment as determined by abdominal computerized tomography scan results. J Urol. 1993:150:874-878.

21. Bahrami A, Ro JY, Ayala AG. An overview of testicular germ cell tumors. Arch Pathol Lab Med. 2007;131:1267-1280.

22. Krag Jacobsen G, Barlebo H, Olsen J, Schultz HP, Starklint $\mathrm{H}$, Søgaard H, et al. Testicular germ cell tumours in Denmark 1976-1980. Pathology of 1058 consecutive cases. Acta Radiol Oncol. 1984;23:239-247.

23. Moul JW, McCarthy WF, Fernandez EB, Sesterhenn IA. Percentage of embryonal carcinoma and of vascular invasion predicts pathological stage in clinical stage I nonseminomatous testicular cancer. Cancer Res. 1994;54:362-364.

24. Vugrin D, Chen A, Feigl P, Laszlo J. Embryonal carcinoma of the testis. Cancer. 1988;61:2348-2352.

25. Leman ES, Gonzalgo ML. Prognostic features and markers for testicular cancer management. Indian J Urol. 2010;26:7681.

26. Mead GM, Stenning SP. The International Germ Cell Consensus Classification: a new prognostic factor-based staging classification for metastatic germ cell tumours. Clin Oncol (R Coll Radiol). 1997;9:207-209.

27. Mirochnik B, Bhargava P, Dighe MK, Kanth N. Ultrasound evaluation of scrotal pathology. Radiol Clin North Am. 2012;50:317-332.

28. vHuang DY, Sidhu PS. Focal testicular lesions: colour Doppler ultrasound, contrast-enhanced ultrasound and tissue elastography as adjuvants to the diagnosis. Br J Radiol. 2012; 85 Spec No 1:S41-S53.

29. Isidori AM, Pozza C, Gianfrilli D, Giannetta E, Lemma A, Pofi R, et al. Differential diagnosis of nonpalpable testicular lesions: qualitative and quantitative contrast-enhanced US of benign and malignant testicular tumors. Radiology. 2014;273:606-618.

30. Westwood M, Joore M, Grutters J, Redekop K, Armstrong N, Lee K, et al. Contrast-enhanced ultrasound using SonoVue ${ }^{\circledR}$ (sulphur hexafluoride microbubbles) compared with contrastenhanced computed tomography and contrast-enhanced magnetic resonance imaging for the characterisation of focal liver lesions and detection of liver metastases: a systematic review and cost-effectiveness analysis. Health Technol Assess. 2013;17:1-243.

31. Schröder C, Lock G, Schmidt C, Löning T, Dieckmann KP. Real-Time Elastography and Contrast-Enhanced Ultrasonography in the Evaluation of Testicular Masses: A Comparative Prospective Study, Ultrasound Med Biol. 2016;42:1807-1815.

32. Radtke A, Cremers JF, Kliesch S, Riek S, Junker K, Mohamed $\mathrm{SA}$, et al. Can germ cell neoplasia in situ be diagnosed by measuring serum levels of microRNA371a-3p? J Cancer Res Clin Oncol. 2017;143:2383-2392.

33. Thornton CP. Best Practice in Teaching Male Adolescents and Young Men to Perform Testicular Self-Examinations: A Review. J Pediatr Health Care. 2016;30:518-527. 